\title{
Review
}

\section{Length of stay, cost, and mortality of healthcare-acquired bloodstream infections in children and neonates: A systematic review and meta-analysis}

\author{
Sofia Karagiannidou MD, MSc ${ }^{1}$, Christos Triantafyllou MSCE ${ }^{1}$, Theoklis E. Zaoutis MD, MSCE, PhD ${ }^{1,2}$, \\ Vassiliki Papaevangelou MD, PhD ${ }^{3}$, Nikolaos Maniadakis BSc, MSc, PhD, FESC ${ }^{4}$ and Georgia Kourlaba MSc, PhD ${ }^{1,5}$ \\ ${ }^{1}$ Center for Clinical Epidemiology and Outcomes Research CLEO, Non-Profit Civil Partnership, Athens, Greece, ${ }^{2}$ Division of Infectious Diseases, The Children's \\ Hospital of Philadelphia, Department of Pediatrics, Philadelphia, Pennsylvania, United States, ${ }^{3}$ Third Department of Pediatrics, National and Kapodistrian \\ University of Athens, School of Medicine, University General Hospital ATTIKON, Athens, Greece, ${ }^{4}$ Department of Health Services Management, National School \\ of Public Health, Athens, Greece and ${ }^{5}$ ECONCARE, LP, Athens, Greece
}

\begin{abstract}
Objective: To estimate the attributable mortality, length of stay (LOS), and healthcare cost of pediatric and neonatal healthcare-acquired bloodstream infections (HA-BSIs).

Design: A systematic review and meta-analysis.

Methods: A systematic search (January 2000-September 2018) was conducted in PubMed, Cochrane, and CINAHL databases. Reference lists of selected articles were screened to identify additional studies. Case-control or cohort studies were eligible for inclusion when full text was available in English and data for at least 1 of the following criteria were provided: attributable or excess LOS, healthcare cost, or mortality rate due to HA-BSI. Study quality was evaluated using the Critical Appraisal Skills Programme Tool (CASP). Study selection and quality assessment were conducted by 2 independent researchers, and a third researcher was consulted to resolve any disagreements. Fixed- or random-effect models, as appropriate, were used to synthesize data. Heterogeneity and publication bias were evaluated.

Results: In total, 21 studies were included in the systematic review and 13 studies were included in the meta-analysis. Attributable mean LOS ranged between 4 and 27.8 days; healthcare cost ranged between $\$ 1,642.16$ and $\$ 160,804$ (2019 USD) per patient with HA-BSI; and mortality rate ranged between $1.43 \%$ and $24 \%$. The pooled mean attributable hospital LOS was 16.91 days (95\% confidence interval [CI], 13.70-20.11) and the pooled attributable mortality rate was $8 \%$ (95\% CI, 6-9). A meta-analysis was not conducted for cost due to lack of eligible studies.

Conclusions: Pediatric HA-BSIs have a significant impact on mortality, LOS, and healthcare cost, further highlighting the need for implementation of HA-BSI prevention strategies.
\end{abstract}

(Received 5 October 2019; accepted 20 November 2019; electronically published 3 January 2020)

Healthcare-associated infections (HAIs) have a significant impact on mortality, length of stay (LOS), and healthcare cost worldwide. ${ }^{1-3}$ In the United States, $\sim 2$ million patients suffer from HAIs annually, nearly 90,000 are estimated to die due to HAIs, and the overall annual direct cost of HAIs to hospitals ranges from $\$ 28$ billion to $\$ 45$ billion. ${ }^{4}$ The 2008 Annual Epidemiological Report on Communicable Diseases in Europe of the European Centre for Disease Prevention and Control (ECDC) declared that HAIs caused 16 million extra days of hospital stay and 37,000 attributable deaths annually, while the associated annual cost reached 7 billion euros (2019 USD, 7.75 billion $)^{5}$

Author for correspondence: S. Karagiannidou, Email: sofiakaragiannidou4@gmail.com Cite this article: Karagiannidou S, et al. (2020). Length of stay, cost, and mortality of healthcare-acquired bloodstream infections in children and neonates: A systematic review and meta-analysis. Infection Control \& Hospital Epidemiology, 41: 342-354, https:// doi.org/10.1017/ice.2019.353
Healthcare-acquired bloodstream infections (HA-BSIs) are the most common HAIs in critically ill pediatric patients of all age groups because central venous catheters (CVCs) are commonly used in these patients. $^{6-9}$ In the United States, HA-BSIs are associated with the highest number of preventable deaths among HAIs, as well as the highest costs, ranging from $\$ 960$ million to $\$ 18.2$ billion annually. ${ }^{10}$ Although strong evidence indicates that most HA-BSIs are preventable, ${ }^{9,11-16}$ pediatric and neonatal HABSI rates remain far above zero in many countries worldwide, highlighting the need to initiate further national and targeted prevention strategies. ${ }^{17-19}$

Published data regarding pediatric and neonatal HA-BSI outcomes vary significantly, depending on the country of origin, year of publication, and study design. ${ }^{20}$ Accurate estimates of LOS, cost, and mortality attributed to HA-BSIs are essential for developing cost-effective infection prevention and infection control measures. ${ }^{21}$ In the present study, we systematically

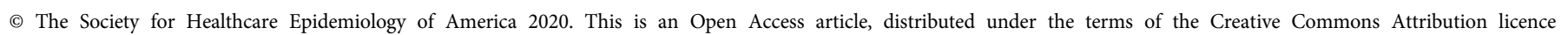
(http://creativecommons.org/licenses/by/4.0/), which permits unrestricted re-use, distribution, and reproduction in any medium, provided the original work is properly cited. 
reviewed the available evidence and estimate attributable LOS, healthcare costs, and mortality rates for pediatric and neonatal HA-BSIs.

\section{Methods}

This study was conducted in accordance with the recommendations of the Preferred Reporting Items for Systematic Reviews and Meta-analyses (PRISMA) statement. ${ }^{22}$ The systematic review protocol is not registered.

\section{Literature search strategy}

A systematic search from January 2000 to September 2018 of the PubMed, Cochrane, and CINAHL databases was conducted by 1 researcher (S.K.) using 3 groups of key words related to the terms "bloodstream," "population," and "outcome." These 3 categories were combined using the Boolean "AND" and "OR." Appendix 1 (online) presents the full search strategy used for MEDLINE, which was adapted for the other databases. Cited references from selected articles were screened to identify additional studies that were not retrieved in the initial search. Conference abstracts were not searched because they do not contain sufficient data for quality assessment.

\section{Selection criteria}

Following the literature search, identified studies were checked to exclude duplicates. The remaining articles were independently screened by 2 researchers (S.K. and C.T.) to identify studies that met the predetermined inclusion criteria. The selection process was performed in 2 steps. In the first step, titles and abstracts were evaluated for eligibility against the predetermined criteria. In the second step, the full-text articles were assessed when the information provided in titles/abstracts was insufficient to decide on inclusion or exclusion. Any disagreements between the 2 researchers were discussed and resolved by a third researcher (G.K.).

The study eligibility criteria were selected by applying the PICOS (population, intervention, comparison, outcomes, and setting) question format:

- Population: Studies referring to neonates and children $<18$ years of age with HA-BSI were eligible, and those that included both adult and pediatric populations were eligible only if stratified results by age group were presented.

- Interventions and comparators: Studies including both a group of children with HA-BSI (BSIs) and a group of children without HA-BSI (non-BSIs) were eligible.

- Outcomes: Studies that provided data on at least 1 of the following factors were included: attributable or excess LOS, or cost, or mortality due to HA-BSIs.

- Study design: Cohorts or case-control studies were selected.

Cohort or case-control study design was set as a criterion because these study types are reported to measure LOS, cost, and mortality more accurately. ${ }^{21}$ Articles that investigated only HA-BSIs caused by specific microorganisms were excluded, as were articles in which the primary outcomes were not the evaluation of attributable or excess LOS, cost, or mortality. Studies that presented mean or median values of the aforementioned attributable outcomes were included in the systematic review; their results are presented separately. However, in the meta-analysis, studies that reported median values of the attributable outcomes were excluded. Finally, only studies with their full text published in English were included.

\section{Data extraction}

Data extraction was performed by 1 researcher (S.K.), and the information was recorded in Microsoft Excel tables (Microsoft, Redmond, WA). The following data were noted: first author, year of publication, country, study design, hospital unit type, definition of HA-BSI, number of children with and without infection (BSIs and non-BSIs), and matching criteria (if used). Moreover, LOS, cost, and mortality (separately for each group), as well as attributable LOS, cost, or mortality, along with the corresponding 95\% confidence intervals, were recorded. In studies where confidence intervals were not provided, we followed the recommendations of the Cochrane collaboration for calculating them. ${ }^{23}$ In cases in which these calculations were not feasible, the relevant studies were excluded from the meta-analysis.

For studies that provided separate estimates for $>1$ hospital unit (eg, the neonatal and the pediatric intensive care units, NICU and PICU), estimates were recorded separately. In 3 studies, the number of children without infection (non-BSI) was calculated by subtracting the BSIs from the total number of pediatric patients included in the study ${ }^{24,25}$ or by applying the matching ratio. ${ }^{26}$

\section{Quality assessment}

Study quality was evaluated by 2 researchers (S.K. and C.T.) using the Critical Appraisal Skills Programme Tool (CASP) for casecontrol and prospective cohort and retrospective cohort studies. ${ }^{27}$ Disagreements were discussed with a third researcher (G.K.), and all 3 researchers ultimately reached consensus.

\section{Statistical analysis}

A meta-analysis was conducted using the STATA commands "metaan" and "metan" to estimate the pooled effect sizes with 95\% confidence intervals (CIs) for attributable LOS and attributable mortality, respectively, as well as to graphically present the results in forest plots. The $\mathrm{I}^{2}$ statistic was used to assess heterogeneity among the included studies. An $\mathrm{I}^{2}>75 \%$ indicates high heterogeneity among studies, and in such case, a random-effects model was used to obtain the pooled effect sizes. Moreover, sensitivity analysis was conducted by removing 1 study each time to identify the study that most influenced the results. Finally, the Egger test and funnel plots were used to evaluate potential publication bias.

\section{Results}

Of the 4,660 papers identified in the literature search, 21 were included in the systematic review $w^{6,24-26,28-44}$ and 13 were included in the meta-analysis (Fig. 1). ${ }^{29,30,32-36,39-44}$

The main characteristics of the participating studies are listed in Table 1. Of the included studies, 4 were conducted in a hematologyoncology unit, ${ }^{6,24,25,44} 6$ involved NICU patients, ${ }^{29,34,38,39,41,42} 6$ involved PICU patients, ${ }^{30,31,35,37,40,43}$ and 5 involved a mixed pediatric population. ${ }^{26,28,32,33,36}$ In most of the studies, ${ }^{24,26,28-32,34-37,39-44}$ HA-BSI was defined according to CDC/NHSN criteria. ${ }^{45-47}$ In 1 study $^{38}$ the Vermont Oxford Network (VON) criteria $^{48,49}$ were used; in another, ICD-9-CM codes were used ${ }^{25}$; and in 2 other studies, institution-based criteria were considered., ${ }^{6,33}$ These institution-based criteria were reasonably chosen by the authors, and they were very much similar to the CDC/NHSN criteria. In addition, 10 of the participating studies were characterized as prospective cohort studies, ${ }^{24,26,28,30,31,35,37,40,41,44} 5$ were characterized as retrospective cohort studies, ${ }^{25,33,36,38,39}$ and 6 were 


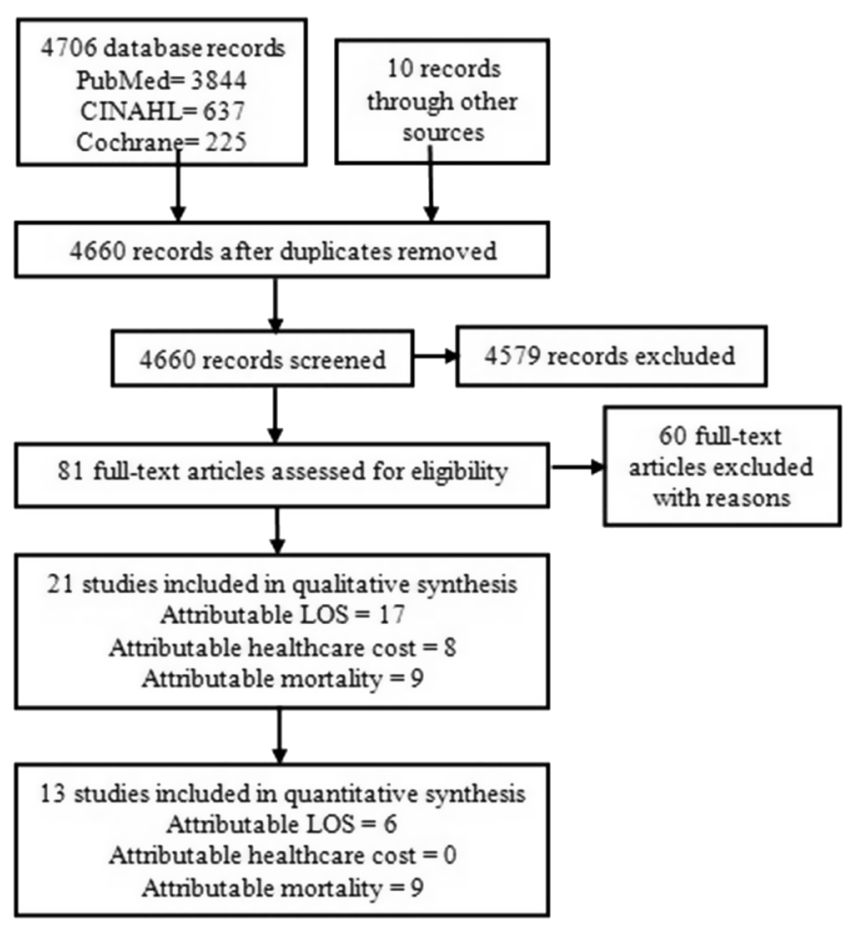

Fig. 1. Flow diagram of included studies. Note. LOS, length of stay.

characterized as case-control studies. ${ }^{6,29,32,34,42,43}$ However, we decided that the included case-control studies should ultimately be classified as either retrospective cohorts ${ }^{6,32,34,42}$ or as prospective cohorts $^{29,43}$ because they actually measured outcomes after prospective or retrospective surveillance of matched BSI and non-BSI children until discharge or death.

Finally, with regard to the methodology of the studies that presented attributable HA-BSI LOS and/or cost, 8 studies used a time-fixed statistical approach ${ }^{25,30,32,37,38,41,43,44}: 7$ of these studies presented time-matched outcomes of BSI and non-BSI patients $6,24,26,28,29,36,39$ and only 1 study used multistate modeling to estimate attributable HA-BSI LOS.33

\section{HA-BSI attributable LOS}

In the systematic review, attributable LOS was presented in 17 studies. ${ }^{6,24-26,28-30,32,33,35-39,41,43,44}$ As shown in Table 2, the attributable mean LOS ranged from 4 to 27.8 days, and in the studies in which median LOS values were presented, attributable median LOS ranged from 1.57 to 12 days. For hospital unit, the attributable mean LOS ranged from 11.4 to 21.1 days in PICUs, from 4 to 27.8 days in NICUs, and from 14.24 to 25.1 days in hematologyoncology units.

In the meta-analysis, 6 studies were included. ${ }^{29,32,35,36,43,44}$ The pooled mean attributable hospital LOS was 16.91 days (95\% CI, 13.70-20.11) (Fig. 2). We detected no heterogeneity amng the studies $\left(\mathrm{I}^{2}=27.74 \%\right)$ and no publication bias (Egger bias test $P=.705$ ) (Appendix 2 online). A subanalysis of 4 studies assessing the attributable LOS of central-line-associated bloodstream infections (CLABSIs), ${ }^{32,35,36,44}$ resulted in a pooled mean attributable LOS of 18.82 days (95\% CI, 15.11-22.54).

For hospital unit, the pooled adjusted mean attributable PICU LOS was 16.4 days ( $95 \%$ CI, $10.1-22.71 ; \mathrm{I}^{2}=0$ ) (Fig. 3a) and the pooled mean attributable NICU LOS was 11.37 days (95\% CI,
4.85-17.89) (Fig. 3b). No heterogeneity was detected between the PICU studies $\left(\mathrm{I}^{2}=0 \%\right)$ and NICU studies $\left(\mathrm{I}^{2}=68.96 \%\right)$. No publication bias was detected either for the PICU studies or the NICU studies: the Egger bias test $P$ statistic was .76 for PICU studies and .07 for NICU studies.

\section{HA-BSI attributable cost}

Attributable healthcare cost was presented in 8 studies, ${ }^{6,25,29,31,32,36,43,44}$ and ranged from $\$ 1,642.16$ to $\$ 160,804$ (2019 USD) per patient with HA-BSI. This range refers to the 7 studies that estimated mean (and not median) attributable healthcare cost (Table 3). At this point, providing specific data around cost per hospital unit would be inaccurate because of the small number of referring studies: only 3 studies measured PICU HA-BSI cost, ${ }^{31,36,43} 2$ studies estimated NICU costs, ${ }^{29,36}$ and 4 studies assessed costs for hematology-oncology patients. ${ }^{6,25,36,44}$ All of these studies differ with regard to the corresponding currency and year.

Meta-analysis was not conducted for the attributable HA-BSI cost due to the lack of eligible studies; only 3 of the participating studies estimated standard error of attributable HA-BSI cost, and their study populations presented heterogeneity. ${ }^{32,36,44}$

\section{HA-BSI attributable mortality}

Attributable mortality was reported in 8 studies ${ }^{30,34,35,39-43}$ and was calculated in 1 study, using estimates that were provided separately for BSI and non-BSI patients. ${ }^{33}$ The attributable mortality rate ranged between 0.01 and 0.24 (Table 4 ). The attributable mortality rate for the NICU was between 0.01 and 0.24 , and for the PICU it was between 0.11 and 0.24 .

In the meta-analysis, 9 studies were included. ${ }^{30,33-35,39-43}$ The pooled mortality rate was 0.08 (95\% CI, 0.06-0.09) (Fig. 4). We detected no statistically significant heterogeneity among the studies $\left(\mathrm{I}^{2}=45.3 \% ; P=.067\right)$. A subanalysis of 4 studies assessing the attributable mortality of CLABSIs $\mathrm{s}^{30,35,40,41}$ resulted in a pooled mean attributable mortality rate of 0.14 (95\% CI, $0.08-0.20)$.

Finally, meta-analysis showed that the pooled attributable mortality rate in NICUs was 0.08 (95\% CI, 0.03-0.13) (Fig. 5) and in PICUs this rate was 0.13 (95\% CI, 0.07-0.19) (Fig. 5). No statistically significant heterogeneity was detected in either NICUs $\left(\mathrm{I}^{2}=58.1 \% ; P=.067\right)$ or PICUs $\left(\mathrm{I}^{2}=0 \% ; P=.76\right)$. As mentioned, no publication bias was detected for either for PICUs or NICUs.

\section{Discussion}

The goal of this systematic review and meta-analysis was to provide evidence around HA-BSI attributable LOS, cost, and mortality among pediatric and neonatal patients, targeting the design and implementation of appropriate and cost-effective prevention strategies. As far as we know, this is the first attempt to synthesize all existing data around HA-BSI outcomes in the pediatric and neonatal population.

The HA-BSI mean attributable LOS ranged from 4 to 27.80 days, and the pooled mean attributable hospital LOS was 16.90 days (95\% CI, 13.70-20.11). Stratified results by type of unit revealed a higher impact of HA-BSIs in PICUs (pooled mean attributable LOS, 16.40 days) compared to NICUs (pooled mean attributable LOS, 11.40 days). The results were more consistent in PICUs, with the mean attributable LOS ranging from 11.40 to 21.10 days, whereas in NICUs it ranged between 4 and 
Table 1. Main Characteristics of the Included Studies

\begin{tabular}{|c|c|c|c|c|c|c|c|c|}
\hline Author (Year) & Country & Study Design & Unit Type & Matching Criteria & BSI Definition & No. of BSIs & No. of Non-BSIs & Outcome \\
\hline Aiken $(2011)^{28}$ & Kenya & $\begin{array}{l}\text { Prospective } \\
\text { cohort }\end{array}$ & $\begin{array}{l}\text { Pediatric ward in } 1 \text { hospital, except for } \\
\text { inpatients with minor trauma or undergoing } \\
\text { elective surgery }\end{array}$ & $\begin{array}{l}\text { 1:4; age, nutritional status, } \\
\text { and LOS at least as long as } \\
\text { the index patient at the point } \\
\text { he or she developed } \\
\text { bacteremia }\end{array}$ & $\begin{array}{l}\text { HA bacteremia, } \\
\text { CDC/NHSN } 2008\end{array}$ & 89 survivors & 353 survivors & LOS \\
\hline Allareddy $(2012)^{25}$ & $\begin{array}{l}\text { United } \\
\text { States }\end{array}$ & $\begin{array}{l}\text { Retrospective } \\
\text { cohort }\end{array}$ & $\begin{array}{l}\text { Hematology-oncology unit, children under } \\
\text { leukemia treatment, Nationwide Inpatient } \\
\text { Sample database, Healthcare and Utilization } \\
\text { Project }\end{array}$ & $\mathrm{N} / \mathrm{A}$ & $\begin{array}{l}\text { Septicemia, identified } \\
\text { using ICD-9-CM codes }\end{array}$ & 787 & 5,433 & $\begin{array}{l}\text { LOS, } \\
\text { cost }\end{array}$ \\
\hline Atif $(2008)^{29}$ & Algeria & $\begin{array}{l}\text { Prospective, } \\
\text { nested case- } \\
\text { control }\end{array}$ & NICU & $\begin{array}{l}\text { 1:2; sex, birth weight, length } \\
\text { of NICU stay } \geq 7 \mathrm{~d} \text {, hospital } \\
\text { admission year }\end{array}$ & $\begin{array}{l}\text { Nosocomial BSI, CDC } \\
1988\end{array}$ & 83 & 166 & $\begin{array}{l}\text { LOS, } \\
\text { cost }\end{array}$ \\
\hline Aviles-Robles $(2014)^{24}$ & Mexico & $\begin{array}{l}\text { Prospective } \\
\text { cohort }\end{array}$ & $\begin{array}{l}\text { Pediatric hematology-oncology unit, children } \\
\text { with febrile neutropenia }\end{array}$ & $\mathrm{N} / \mathrm{A}$ & LCBI, CDC/NHSN 2008 & 24 & 193 & LOS \\
\hline Biwersi $(2009)^{6}$ & Germany & Case-control & Pediatric hematology-oncology unit & $\begin{array}{l}\text { 1:1; age, gender, underlying } \\
\text { malignancy, chemotherapy, } \\
\text { exact date of event }\end{array}$ & Institution-based criteria & 43 & 43 & $\begin{array}{l}\text { LOS, } \\
\text { cost }\end{array}$ \\
\hline Dramowski $(2016)^{26}$ & $\begin{array}{l}\text { South } \\
\text { Africa }\end{array}$ & $\begin{array}{l}\text { Prospective } \\
\text { cohort }\end{array}$ & $\begin{array}{l}\text { General pediatrics, pediatric surgery, pediatric } \\
\text { infectious diseases/gastroenterology/ } \\
\text { cardiology, and PICU }\end{array}$ & $\begin{array}{l}\text { 1:3; Age- and ward-matched } \\
\text { controls per HCAI event, } \\
\text { hospitalized for at least as } \\
\text { long as the index patient }\end{array}$ & LCBI, CDC/NHSN 2013 & 41 & 123 & LOS \\
\hline Duenas $(2011)^{30}$ & $\begin{array}{l}\text { INICC, El } \\
\text { Salvador }\end{array}$ & $\begin{array}{l}\text { Prospective } \\
\text { cohort }\end{array}$ & PICU & N/A & CLABSI, CDC/NHSN 2008 & 40 & 994 & $\begin{array}{l}\text { LOS, } \\
\text { mortality }\end{array}$ \\
\hline Elward $(2005)^{31}$ & $\begin{array}{l}\text { United } \\
\text { States }\end{array}$ & $\begin{array}{l}\text { Prospective } \\
\text { cohort }\end{array}$ & PICU & N/A & $\begin{array}{l}\text { Nosocomial primary BSI, } \\
\text { CDC/NHSN } 1996\end{array}$ & 57 & 854 & Cost \\
\hline Goudie $(2014)^{32}$ & $\begin{array}{l}\text { United } \\
\text { States }\end{array}$ & Case-control & $\begin{array}{l}\text { Children with inpatient discharges in the } \\
\text { Nationwide Inpatient Sample databases, } \\
\text { Healthcare and Utilization Project }\end{array}$ & $\begin{array}{l}\text { 1:2; age, discharge year, and } \\
\text { propensity score }\end{array}$ & CLABSI, CDC/NHSN 2012 & 1,339 & 2,678 & $\begin{array}{l}\text { LOS, } \\
\text { cost }\end{array}$ \\
\hline Green $(2014)^{33}$ & England & $\begin{array}{l}\text { Retrospective } \\
\text { cohort }\end{array}$ & $\begin{array}{l}\text { National laboratory surveillance database } \\
\text { (LabBase2), including NICUs, PICUs, General } \\
\text { and Specialist Pediatric Medical/Surgical } \\
\text { wards }\end{array}$ & $\mathrm{N} / \mathrm{A}$ & $\begin{array}{l}\text { Institution- based } \\
\text { criteria }\end{array}$ & 214 & 333,391 & $\begin{array}{l}\text { LOS, } \\
\text { mortality }\end{array}$ \\
\hline Grisaru-Soen $(2012)^{34}$ & Israel & $\begin{array}{l}\text { Retrospective } \\
\text { matched } \\
\text { case-control }\end{array}$ & NICU & $\begin{array}{l}\text { 1:1; gestational age, birth } \\
\text { weight, and duration } \\
\text { of NICU hospitalization prior } \\
\text { to the onset of } \\
\text { late-onset sepsis. }\end{array}$ & $\begin{array}{l}\text { Nosocomial late-onset } \\
\mathrm{BSI}, \mathrm{CDC} / \mathrm{NHSN} 2008\end{array}$ & 101 & 101 & Mortality \\
\hline Gupta $(2011)^{35}$ & India & $\begin{array}{l}\text { Prospective } \\
\text { surveillance }\end{array}$ & PICU & $\mathrm{N} / \mathrm{A}$ & $\begin{array}{l}\text { HA-BSI, CVC-BSI, CDC/ } \\
\text { NHSN } 2008\end{array}$ & $\begin{array}{l}11 \mathrm{HA}-\mathrm{BSI} ; 11 \\
\text { CVC-BSI }\end{array}$ & $\begin{array}{l}176 \text { no HA-BSI; } 120 \\
\text { no CVC-BSI }\end{array}$ & $\begin{array}{l}\text { LOS, } \\
\text { mortality }\end{array}$ \\
\hline $\begin{array}{l}\text { Karagiannidou } \\
(2018)^{36}\end{array}$ & Greece & $\begin{array}{l}\text { Retrospective } \\
\text { cohort }\end{array}$ & $\begin{array}{l}\text { PICU, NICU, hematology-oncology unit, and } \\
\text { bone marrow transplantation unit }\end{array}$ & $\begin{array}{l}1: 1 ; \text { hospital, unit, and length } \\
\text { of stay prior to study } \\
\text { enrollment }\end{array}$ & CLABSI, CDC/NHSN 2014 & 94 & 94 & $\begin{array}{l}\text { LOS, } \\
\text { cost }\end{array}$ \\
\hline
\end{tabular}


Table 1. (Continued)

\begin{tabular}{|c|c|c|c|c|c|c|c|c|}
\hline Author (Year) & Country & Study Design & Unit Type & Matching Criteria & BSI Definition & No. of BSIs & No. of Non-BSIs & Outcome \\
\hline Navoa-Ng $(2011)^{37}$ & $\begin{array}{l}\text { INICC, } \\
\text { Philippines }\end{array}$ & $\begin{array}{l}\text { Prospective } \\
\text { cohort }\end{array}$ & PICU & $\mathrm{N} / \mathrm{A}$ & CLABSI, CDC/NHSN 2008 & 4 & 240 & LOS \\
\hline Payne $(2004)^{38}$ & $\begin{array}{l}\text { United } \\
\text { States }\end{array}$ & $\begin{array}{l}\text { Retrospective } \\
\text { cohort }\end{array}$ & NICU & $\mathrm{N} / \mathrm{A}$ & $\begin{array}{l}\text { Nosocomial BSI (NBI), } \\
\text { Vermont Oxford Network } \\
\text { (VON) criteria } 1998 \text { and } \\
1999\end{array}$ & $\begin{array}{l}553 ; \text { CONS, 372; } \\
\text { other bacteria, } \\
\text { 232; fungemia, } \\
85\end{array}$ & 2,256 & LOS \\
\hline Pessoa-Silva $(2001)^{39}$ & Brazil & $\begin{array}{l}\text { Retrospective } \\
\text { matched } \\
\text { cohort }\end{array}$ & NICU & $\begin{array}{l}\text { 1:1; sex, birth weight, } \\
\text { gestational age, secondary } \\
\text { diagnosis, hospitalization for } \\
\text { at least as long as the } \\
\text { matched case before } \\
\text { the LO-BSI event }\end{array}$ & $\begin{array}{l}\text { Nosocomial Late-onset } \\
\text { BSI, CDC/NHSN } 1988\end{array}$ & 50 & 50 & $\begin{array}{l}\text { LOS, } \\
\text { mortality }\end{array}$ \\
\hline Rosenthal $(2014)^{41}$ & $\begin{array}{l}\text { INICC, } 43 \\
\text { countries }\end{array}$ & $\begin{array}{l}\text { Prospective } \\
\text { cohort }\end{array}$ & NICU & N/A & CLABSI, CDC/NHSN 2008 & 51 & 7,447 & $\begin{array}{l}\text { LOS, } \\
\text { mortality }\end{array}$ \\
\hline Rosenthal $(2012)^{40}$ & $\begin{array}{l}\text { INICC, } 16 \\
\text { limited- } \\
\text { resource } \\
\text { countries }\end{array}$ & $\begin{array}{l}\text { Prospective } \\
\text { cohort }\end{array}$ & PICU & N/A & CLABSI, CDC/NHSN 2008 & 95 & 3,285 & Mortality \\
\hline Schwab $(2015)^{42}$ & Germany & $\begin{array}{l}\text { Retrospective } \\
\text { cohort and } \\
\text { case-control }\end{array}$ & NICU, very low birth weight infants (VLBW) & $\begin{array}{l}\text { Case-control study, } 1: 1 ; \\
\text { department, gestational age, } \\
\text { length of stay of the control } \\
\text { >length of stay to the start of } \\
\text { infection of the case, start of } \\
\text { surveillance, sex, mode of } \\
\text { delivery }\end{array}$ & $\begin{array}{l}\text { BSI and laboratory } \\
\text { confirmed (LC-BSI, no } \\
\text { CoNS) CDC/ NHSN } 2008\end{array}$ & $\begin{array}{l}\text { Cohort: } \\
\text { 6,911 BSIs; } \\
\text { 1,806 LC-BSIs } \\
\text { Case-control: } \\
\text { 5,187 BSIs; } \\
\text { 1,511 LC-BSIs }\end{array}$ & $\begin{array}{l}\text { Cohort: } \\
\text { 36,205 no BSIs; } \\
41,310 \text { no LC-BSIs; } \\
\text { Case-control: } \\
\text { 5,187 non-BSIs; } \\
\text { 1,511 non-LC-BSIs }\end{array}$ & Mortality \\
\hline Slonim $(2001)^{43}$ & $\begin{array}{l}\text { United } \\
\text { States }\end{array}$ & Case-control & PICU & $\begin{array}{l}\text { 1:1; age, severity of illness, } \\
\text { primary diagnosis, and } \\
\text { admission date }\end{array}$ & $\begin{array}{l}\text { Nosocomial BSI, CDC/ } \\
\text { NHSN } 1988\end{array}$ & 38 & 38 & $\begin{array}{l}\text { LOS, } \\
\text { cost, } \\
\text { mortality }\end{array}$ \\
\hline Wilson $(2014)^{44}$ & $\begin{array}{l}\text { United } \\
\text { States }\end{array}$ & $\begin{array}{l}\text { Prospective } \\
\text { cohort }\end{array}$ & Pediatric hematology-oncology unit & 1:1; propensity score & CLABSI, CDC/NHSN 2014 & 59 & 59 & $\begin{array}{l}\text { LOS, } \\
\text { cost }\end{array}$ \\
\hline
\end{tabular}

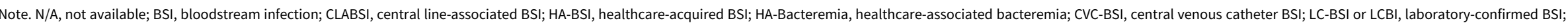

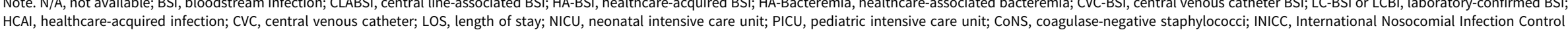
Consortium; CDC, Centers for Disease Control and Prevention; NHSN, National Healthcare Safety Network. 
Table 2. Outcome Data of Included Studies With Regard to Length of Stay (LOS)

\begin{tabular}{|c|c|c|c|c|}
\hline $\begin{array}{l}\text { Author } \\
\text { (Year) }\end{array}$ & Adjustment & BSI LOS, d & Non-BSI LOS, d & Attributable LOS, d \\
\hline $\begin{array}{l}\text { Aiken } \\
(2011)^{28}\end{array}$ & A confounder and time matching approach & $\begin{array}{l}\text { Median LOS of matched } \\
\text { survivors, } 28.9 \\
\text { (IQR, 13-41.6) }\end{array}$ & $\begin{array}{l}\text { Median LOS of matched } \\
\text { survivors, } 18.8 \\
\text { (IQR, 8.1-30.9) }\end{array}$ & Additional attributable LOS, 10.1 \\
\hline $\begin{array}{l}\text { Allareddy } \\
(2012)^{25}\end{array}$ & $\begin{array}{l}\text { Presence of septicemia, age, gender, patient disposition, insurance status, } \\
\text { leukemia type, admission type, presence of comorbid conditions, and hospital } \\
\text { teaching status, location, geographic region, and bed size. }\end{array}$ & Average (crude), 33.18 & Average (crude), 13.79 & Estimated adjusted increase in LOS, 14.24 \\
\hline $\begin{array}{l}\text { Atif } \\
(2008)^{29}\end{array}$ & N/A & $\begin{array}{l}\text { Mean in NICU, } 24.3 \\
(S D, \pm 18.7)\end{array}$ & $\begin{array}{l}\text { Mean in NICU, } 15.1 \\
(\text { SD, } \pm 11.3)\end{array}$ & Mean attributable extra LOS, 9.2 \\
\hline $\begin{array}{l}\text { Aviles-Robles } \\
(2014)^{24}\end{array}$ & Age at admission, sex, cancer type, and antimicrobial prophylaxis & Crude median, 19 & Crude median, 10 & $\begin{array}{l}\text { Patients with BSIs had a } 100 \% \text { longer } \\
\text { relative LOS, compared with patients for } \\
\text { whom no pathogen was identified }\end{array}$ \\
\hline $\begin{array}{l}\text { Biwersi } \\
(2009)^{6}\end{array}$ & $\mathrm{~N} / \mathrm{A}$ & $\begin{array}{l}\text { Median inpatient antimicrobial } \\
\text { treatment, } 12 \\
\text { (IQR, 11-18; range, 7-59) }\end{array}$ & $\begin{array}{l}\text { Median inpatient } \\
\quad \text { antimicrobial } \\
\text { treatment, } 0 \\
\text { (IQR, 0-7; range, 0-34) }\end{array}$ & $\begin{array}{l}\text { Median additional length of inpatient } \\
\text { antimicrobial treatment, } 12 \\
\text { (IQR, 8.5-16) }\end{array}$ \\
\hline $\begin{array}{l}\text { Dramowski } \\
(2016)^{26}\end{array}$ & $\begin{array}{l}\text { A confounder and time-matching approach was used, after excluding patient } \\
\text { admission episodes with outcome of death or transfer }\end{array}$ & $\begin{array}{l}\text { Crude median, } 20 \\
\text { (IQR, 11-32) }\end{array}$ & $\begin{array}{l}\text { Crude median, } 11 \\
\text { (IQR, 7-23) }\end{array}$ & Median excess LOS, 9 \\
\hline $\begin{array}{l}\text { Duenas } \\
(2011)^{30}\end{array}$ & N/A & Average, 19.1 & Average, 6.2 & Extra LOS, 12.9 \\
\hline $\begin{array}{l}\text { Goudie } \\
(2014)^{32}\end{array}$ & $\begin{array}{l}\text { CLABSI status, age, discharge year, gender, race/ethnicity, insurance status, } \\
\text { propensity score, and interactions for CLABSI by age, gender, race/ethnicity, } \\
\text { insurance category and year }\end{array}$ & Mean adjusted, 37.2 & Mean adjusted, 18.2 & $\begin{array}{l}\text { Mean adjusted attributable, } 19 \\
(95 \% \mathrm{Cl}, 14.3-23.8)\end{array}$ \\
\hline $\begin{array}{l}\text { Green } \\
(2014)^{33}\end{array}$ & $\begin{array}{l}\text { Time-adjusted estimate of excess LOS, weighted relative to the frequency of } \\
\text { transitions to HA-BSI, discharge alive or in-hospital death on each day. }\end{array}$ & $\begin{array}{l}\text { Crude mean, 56.55; median, } 30 \\
\text { (IQR, 10-66) }\end{array}$ & $\begin{array}{l}\text { Crude mean, } 1.35 \\
\text { median, } 0.5 \text { (IQR, } 0.5-1)\end{array}$ & $\begin{array}{l}\text { Adjusted excess LOS, } 1.57 \\
(95 \% \mathrm{Cl}, 0.20-2.95)\end{array}$ \\
\hline $\begin{array}{l}\text { Gupta } \\
(2011)^{35}\end{array}$ & $\mathrm{~N} / \mathrm{A}$ & Mean, 25.6 (SE, 7.2) & Mean, 9.6 (SE, 0.87) & $\begin{array}{l}\text { Mean attributable LOS, } 16 \\
\text { (SE, 4.3) }\end{array}$ \\
\hline $\begin{array}{l}\text { Karagiannidou } \\
(2018)^{36}\end{array}$ & $\begin{array}{l}\text { Age, gender, matching characteristics, central line management after study } \\
\text { enrollment, and propensity score for predicting the risk of acquiring a CLABSI. }\end{array}$ & $\begin{array}{l}\text { Mean crude, } 55.2 \\
\text { (95\% CI, 44.8-66.5) } \\
\text { Mean adjusted, 58.7 } \\
\text { Mean adjusted by unit: PICU, 55; } \\
\text { NICU, 75.5; hematology- } \\
\text { oncology, 71; BMTU, } 33.9\end{array}$ & $\begin{array}{l}\text { Mean crude, } 39.7 \\
\text { (95\% CI, 31.4-49.6) } \\
\text { Mean adjusted, } 37.6 \\
\text { Mean adjusted by unit: } \\
\text { PICU, 35.2; NICU, } \\
\text { 48.3; hematology- } \\
\text { oncology unit, 45.5; } \\
\text { BMTU, 21.7 }\end{array}$ & $\begin{array}{l}\text { Mean adjusted attributable, } 21.3 \\
\quad(95 \% \mathrm{Cl}, 6.8-35.8) \\
\text { Mean adjusted attributable by unit: } \\
\text { PICU, } 20(95 \% \mathrm{Cl}, 3.9-36.2) ; \\
\text { NICU, } 27.8(95 \% \mathrm{Cl}, 8-47.5) ; \\
\text { hematology-oncology unit, } 25.1 \\
\quad(95 \% \mathrm{Cl}, 7.9-42.3) \text {; } \\
\text { BMTU, } 12.3(95 \% \mathrm{Cl}, 4.5-20.1)\end{array}$ \\
\hline $\begin{array}{l}\text { Navoa-Ng } \\
(2011)^{37}\end{array}$ & N/A & Average, 17 & Average, 5.6 & $\begin{array}{l}\text { Crude extra LOS, } 11.4 \\
(95 \% \mathrm{Cl}, 6.9-62.5)\end{array}$ \\
\hline
\end{tabular}


Table 2. (Continued)

\begin{tabular}{|c|c|c|c|c|}
\hline $\begin{array}{l}\text { Author } \\
\text { (Year) }\end{array}$ & Adjustment & BSI LOS, d & Non-BSI LOS, d & Attributable LOS, $d$ \\
\hline $\begin{array}{l}\text { Payne } \\
(2004)^{38}\end{array}$ & $\begin{array}{l}\text { Birth weight, small for gestational age, birth location, gender, maternal race, } \\
\text { prenatal care, antenatal steroids, multiple birth, 5-min APGAR score, respiratory } \\
\text { distress syndrome, chronic lung disease, necrotizing enterocolitis (NEC), NEC } \\
\text { surgery, other surgery, and any ventilation }\end{array}$ & $\begin{array}{l}\text { Crude mean LOS per BW } \\
\text { category: } \\
\text { BW } 401-750 \mathrm{~g}, 101 \\
\text { BW } 751-1,000 \mathrm{~g}, 83 \\
\text { BW } 1,001-1,250 \mathrm{~g}, 60 \\
\text { BW } 1,251-1,500 \mathrm{~g}, 48 \\
\text { Adjusted mean LOS: } \\
\text { BW } 401-750 \mathrm{~g}, 94 \\
\text { BW } 751-1,000 \mathrm{~g}, 75 \\
\text { BW } 1001-1,250 \mathrm{~g}, 51 \\
\text { BW } 1,251-1,500 \mathrm{~g}, 36\end{array}$ & $\begin{array}{l}\text { Crude mean LOS per } \\
\text { BW category: } \\
\text { BW } 401-750 \mathrm{~g}, 85 \\
\text { BW } 751-1,000 \mathrm{~g}, 66 \\
\text { BW } 1,001-1,250 \mathrm{~g}, 47 \\
\text { BW } 1,251-1,500 \mathrm{~g}, 32 \\
\text { Adjusted mean LOS: } \\
\text { BW } 401-750 \mathrm{~g}, 88 \\
\text { BW } 751-1,000 \mathrm{~g}, 68 \\
\text { BW } 1,001-1,250 \mathrm{~g}, 47 \\
\text { BW } 1,251-1,500 \mathrm{~g}, 31\end{array}$ & $\begin{array}{l}\text { Crude mean excess LOS per BW category: } \\
\text { BW 401-750 g, } 16 \\
\text { BW 751-1,000 g, } 17 \\
\text { BW 1,001-1,250 g, } 13 \\
\text { BW 1,251-1,500 g, } 16 \\
\text { Adjusted mean excess LOS: } \\
\text { BW 401-750 g, } 6 \\
\text { BW 751-1,000 g, } 7 \\
\text { BW 1,001-1,251 g, } 4 \\
\text { BW 1,251-1,500 g, } 5\end{array}$ \\
\hline $\begin{array}{l}\text { Pessoa-Silva } \\
(2001)^{39}\end{array}$ & $\begin{array}{l}\text { Nasogastric/tracheal tube, lumbar/arterial puncture, thoracic drains, blood and/or } \\
\text { blood components transfusion, umbilical catheter, parenteral nutrition, duration of } \\
\text { peripheral/central venous catheters, and antibiotics }\end{array}$ & $\begin{array}{l}\text { Mean, } 57.6 \text {; } \\
\text { In } 32 \text { pairs where both } \\
\text { subjects survived: } \\
\text { mean, } 53.2\end{array}$ & $\begin{array}{l}\text { Mean, } 43.6 \\
\text { In } 32 \text { pairs where both } \\
\text { subjects survived: } \\
\text { mean, } 28.1\end{array}$ & $\begin{array}{l}\text { In } 32 \text { pairs where both subjects survived: } \\
\text { prolongation of LOS, } 25.1\end{array}$ \\
\hline $\begin{array}{l}\text { Rosenthal } \\
(2014)^{41}\end{array}$ & $\mathrm{~N} / \mathrm{A}$ & $\begin{array}{l}\text { Pooled average, } 23.22 \\
(95 \% \mathrm{Cl}, 17.78-31.03)\end{array}$ & $\begin{array}{l}\text { Pooled average, } 10.75 \\
(95 \% \mathrm{Cl}, 10.53-10.99)\end{array}$ & Pooled average extra, 12.46 \\
\hline $\begin{array}{l}\text { Slonim } \\
(2001)^{43}\end{array}$ & $\mathrm{~N} / \mathrm{A}$ & $\begin{array}{l}\text { Mean PICU LOS, } 19.3 \\
(\text { SEM, } \pm 2.3 \text { ) } \\
\text { Mean hospital LOS, } 46.7 \\
(\text { SEM, } \pm 4.9)\end{array}$ & $\begin{array}{l}\text { Mean PICU LOS, } 4.6 \\
(\text { SEM, } \pm 6.5 \text { ) } \\
\text { Mean hospital LOS, } 24.4 \\
\text { (SEM, } \pm 6.5 \text { ) }\end{array}$ & $\begin{array}{l}\text { Attributable PICU LOS, } 14.6 \\
\text { Attributable hospital LOS, } 21.1\end{array}$ \\
\hline $\begin{array}{l}\text { Wilson } \\
(2014)^{44}\end{array}$ & $\begin{array}{l}\text { Age, sex, central venous catheter type, total no. of line days, the no. of times the } \\
\text { CVC was accessed by hospital personnel other than those on the pediatric } \\
\text { hematology-oncology ward, no. of blood cultures and the total no. of excessive } \\
\text { blood cultures }\end{array}$ & Mean, 33.9 & Mean, 12.7 & $\begin{array}{l}\text { Attributable, } 21.2 \\
(95 \% \mathrm{Cl}, 10.4-32)\end{array}$ \\
\hline
\end{tabular}

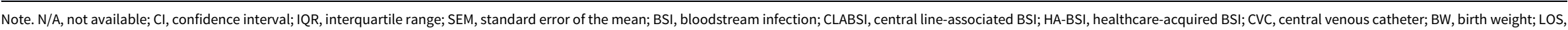
length of stay; NICU, neonatal intensive care unit; PICU, pediatric intensive care unit; BMTU, bone marrow transplantation unit. 


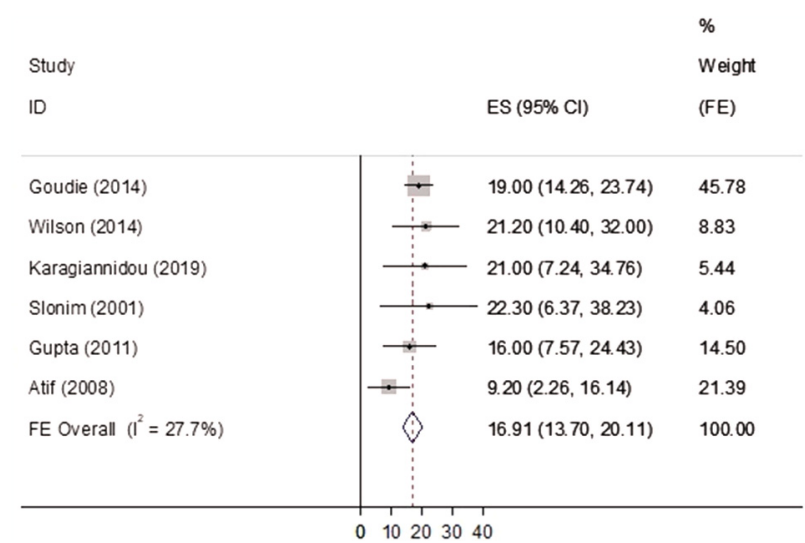

Fig. 2. Pooled mean of attributable hospital length of stay (LOS), forest plot. Note. ES, effect size; FE, fixed effects.

(a)

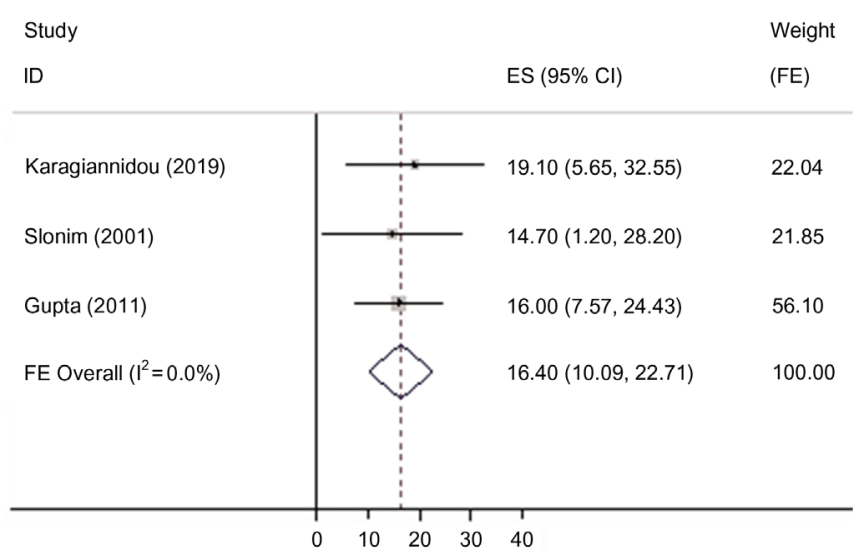

(b)

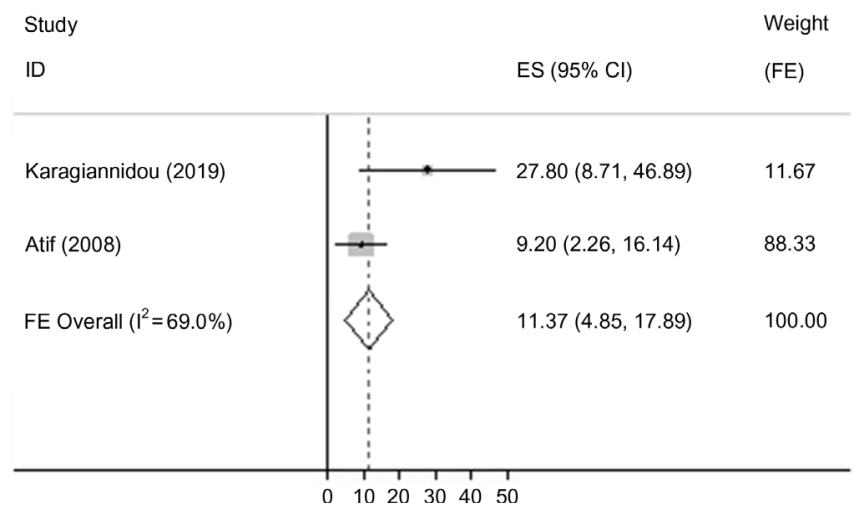

Fig. 3. (a) Pooled mean of attributable hospital length of stay (LOS) in the pediatric intensive care unit (PICU), forest plot; (b) Pooled mean of attributable hospital length of stay (LOS) in the neonatal intensive care unit (NICU), forest plot. Note. ES, effect size; $F E$, fixed effects.

27.80 days. However, no statistically significant heterogeneity was observed in PICUs or in NICUs.

As described in the previous section, 7 of the studies that participated in the meta-analysis presented time-matched outcomes of BSI and non-BSI patients with regard to LOS, and only one used a multistate modeling approach to estimate attributable HA-BSI LOS. Previous work by Manoukian et $\mathrm{al}^{21}$ has suggested that excess LOS associated with HA-BSIs in adult populations presents significant variations according to the statistical method employed. More specifically, Manoukian et al suggest that studies using time-fixed methods overestimate the attributable LOS compared to timevarying methods, ${ }^{21}$ because they do not take into account the time-dependent bias. Multistate modeling is considered the most accurate statistical method of attributable LOS estimation..$^{20,50}$ This hypothesis can only be partially confirmed by our study because the only study using multistate modeling among those included in our review provided the lower estimation of excess LOS (1.57 days)..$^{33}$

Attributable mean healthcare cost ranged from $\$ 1,642.16$ to $\$ 160,804$ (2019 USD) per patient with HA-BSI. Previous work by Umscheid et $\mathrm{al}^{10}$ assessed attributable cost of catheterassociated BSIs (CA-BSIs) in the adult ICU and reported costs from $\$ 41,900$ to $\$ 123,600$ (2009 USD).

In general, the large difference observed between reported attributable cost estimates for several HAI types is due to differences in the perspective of cost analysis (ie, hospital or societal), the costing methodology (ie, microcosting approach or not), and the year of costing, as well as differences in clinical practice patterns and healthcare systems among countries (ie, use of novel and expensive technologies in high-income countries, etc). ${ }^{20}$

Attributable mortality rate ranged between 0.01 and 0.24 , and pooled mortality was 0.08 (95\% CI, 0.06-0.09). Stratified analysis by type of unit revealed that the pooled mortality rate was higher in PICUs (0.13) compared to NICUs (0.08).

Previous systematic reviews and meta-analyses have presented data indicating that the odds ratio for in-hospital death associated with HA-BSIs in adult patients ranges between 1.96 and $2.75 .^{51,52}$ However, attributable HA-BSI mortality rates present significant variations, according to several causative microorganisms and susceptibility patterns. ${ }^{51,53}$

Quantifying excess HA-BSI outcomes is essential for both healthcare providers and policy makers. Improving efficiency with regard to resources and bed days by implementing targeted prevention strategies is crucial to increasing a hospital's capacity to provide high-quality care to the highest number of patients. Precise measurements of HA-BSI outcomes could guide decision making around investments in infection control.

This study has several limitations. First, we acknowledge the possibility of language bias due to the fact that only studies written in English were incorporated in this review. Practical reasons, namely the difficulty of translating from a variety of languages, led us to the decision to include only English-language studies. Moreover, restricting the search strategy to only electronic databases may have introduced publication bias because this approach is unlikely to identify studies that have not been published in peerreviewed journals. Because we did not include unpublished studies in our review, it was impossible to assess the potential publication bias by comparing the results of published and unpublished studies. However, we applied the Egger test, which revealed that no publication bias exists in HA-BSI-attributable LOS and mortality studies. We should underscore that the Egger test is inappropriate where there is heterogeneity; the test has low power and is of little use in analyses with few studies.

Finally, another important limitation of this study is the inclusion of studies that used a variety of HA-BSI definitions, and 


\begin{tabular}{|c|c|c|c|c|}
\hline Author (Year) & Adjustment & BSI Costs & Non-BSI Costs & Attributable Costs \\
\hline Allareddy $(2012)^{25}$ & $\begin{array}{l}\text { Presence of septicemia, age, gender, patient disposition, insurance status, } \\
\text { leukemia type, admission type, presence of comorbid conditions, and hospital } \\
\text { teaching status, location, geographic region, and bed size }\end{array}$ & $\begin{array}{l}\text { Mean (crude) hospitalization } \\
\text { charges, } \$ 279,137 \\
\text { (2019 USD: } \$ 334,276.74)\end{array}$ & $\begin{array}{l}\text { Mean (crude) hospitalization } \\
\quad \text { charges, } \$ 113,530 \\
\text { (2019 USD, \$135,956.32) }\end{array}$ & $\begin{array}{l}\text { Estimated adjusted increase in } \\
\text { hospitalization charges (2008 USD), } \\
\quad \$ 134,279 \\
(2019 \text { USD, } \$ 160,804)\end{array}$ \\
\hline Atif $(2008)^{29}$ & $\mathrm{~N} / \mathrm{A}$ & $\begin{array}{l}\text { Mean cumulative, } \$ 2584 \\
\text { (2019 USD, } \$ 3,226.89)\end{array}$ & $\begin{array}{l}\text { Mean cumulative, } \$ 1,269 \\
\text { (2019 USD, \$1584.72) }\end{array}$ & $\begin{array}{l}\text { Mean cumulative attributable extra, } \\
\$ 1,315 \\
(2019 \text { USD, } \$ 1,642.16)\end{array}$ \\
\hline Biwersi $(2009)^{6}$ & $\mathrm{~N} / \mathrm{A}$ & N/A & N/A & $\begin{array}{l}\text { Median additional expenses, } € 4,400 \\
\quad(\text { IQR, 3,145-5,920) } \\
\text { or } \$ 6,970(\text { IQR, } 4,938-9,294) \\
(2019 \text { USD, } \$ 8,304)\end{array}$ \\
\hline Elward $(2005)^{31}$ & $\begin{array}{l}\text { Nosocomial primary BSI, age, severity of illness, underlying disease (congenital } \\
\text { heart disease/transplant), and ventilator days }\end{array}$ & $\begin{array}{l}\text { Adjusted mean direct costs of } \\
\text { PICU admission, } \$ 45,615 \\
\text { (2019 USD, } \$ 67,728)\end{array}$ & $\begin{array}{l}\text { Adjusted mean direct costs } \\
\text { of PICU admission, } \$ 6,396 \\
(2019 \text { USD, } \$ 9,496)\end{array}$ & $\begin{array}{l}\text { Adjusted attributable mean direct cost } \\
\text { of PICU admission (1999 and } 2000 \\
\text { USD), \$39,219 } \\
\text { (2019 USD, \$58,231) }\end{array}$ \\
\hline Goudie $(2014)^{32}$ & $\begin{array}{l}\text { CLABSI status, age, discharge year, gender, race/ethnicity, insurance status, } \\
\text { propensity score and interactions for CLABSI by age, gender, race/ethnicity, } \\
\text { insurance category and year }\end{array}$ & $\begin{array}{l}\text { Mean adjusted, } \$ 103,949 \\
\text { (2019 USD, } \$ 119,314.57)\end{array}$ & $\begin{array}{l}\text { Mean adjusted, } \$ 48,303 \\
\text { (2019 USD, } \$ 55,443.07)\end{array}$ & $\begin{array}{l}\text { Mean adjusted attributable (2011 USD), } \\
\quad \$ 55,646 \\
(95 \% \mathrm{Cl}, 38,785-72,507) \\
{[2019 \text { USD, } \$ 63,871.5(\mathrm{SE}, 9,874)]}\end{array}$ \\
\hline $\begin{array}{l}\text { Karagiannidou } \\
(2018)^{36}\end{array}$ & $\begin{array}{l}\text { Age, gender, matching characteristics, central line management after study } \\
\text { enrollment, and propensity score for predicting the risk of acquiring a CLABSI. }\end{array}$ & $\begin{array}{l}\text { Mean adjusted, } € 31,944 \\
\text { Mean adjusted cost by unit: } \\
\text { PICU, } € 31,192 ; \\
\text { NICU, } € 36,791 ; \\
\text { Hematology-oncology unit, } \\
\quad € 39,431 ; \\
\text { BMTU, } € 21,157 \\
\text { (2019 USD: } \\
\text { Overall adjusted, } \$ 56,490 ; \\
\text { PICU, } \$ 53,830 ; \\
\text { NICU, } \$ 66,019 ; \\
\text { hematology-oncology unit, } \\
\$ 69,486 ; \\
\text { BMTU, } \$ 38,198)\end{array}$ & $\begin{array}{l}\text { Mean adjusted, } € 18,072 \\
\text { Mean adjusted cost by unit: } \\
\text { PICU, } € 17,646 \text {; } \\
\text { NICU, } € 20814 \text {; hematology- } \\
\text { oncology unit, } € 22307 ; \\
\text { BMTU, } € 11,969 \\
\text { (2019 USD: } \\
\text { Overall adjusted, \$17,788; } \\
\text { PICU, \$16,951; } \\
\text { NICU, \$20,788; } \\
\text { hematology-oncology unit, } \\
\quad \$ 21,881 ; \\
\text { BMTU, } \$ 12,028 \text { ) }\end{array}$ & $\begin{array}{l}\text { Mean adjusted attributable, } € 14,099 \\
\text { ( } 95 \% \mathrm{Cl}, 5,631-22,568) \\
\text { Mean adjusted attributable by unit } \\
\quad(2017 €) \text { : } \\
\text { PICU, } € 13,852 \\
\text { (95\% CI, 5,409-22,296); } \\
\text { NICU, } € 16,493 \\
\text { (95\% Cl, 5,623-27,363) hematology- } \\
\text { oncology unit, } € 16,934 \\
\text { (95\% Cl, 5,599-28,269); } \\
\text { BMTU, } € 9,320 \\
\text { (95\% CI, 4,099-14,541) } \\
\text { [2019 USD: } \\
\text { Overall, \$24,773 (SE, 7337); } \\
\text { PICU, \$23,748 (SE, 7,842); } \\
\text { NICU, \$29,371 (SE, 9,282); hematology- } \\
\text { oncology unit, \$29,673 (SE, 9,940); } \\
\text { BMTU, \$16,733 (SE, 5,166)] }\end{array}$ \\
\hline $\begin{array}{l}\text { Slonim } \\
(2001)^{43}\end{array}$ & $\mathrm{~N} / \mathrm{A}$ & $\begin{array}{l}\text { Mean total operational costs, } \\
\quad \$ 78,272 \text { (SEM, } \pm 8,202) \\
{[2019 \text { USD, } \$ 117,211.39 \text { (SE, }} \\
12,282.40)]\end{array}$ & $\begin{array}{l}\text { Mean total operational costs, } \\
\quad \$ 35,005 \\
(\text { SEM, } \pm 9,865) \\
{[2019 \text { USD, } \$ 52,419.57 \text { (SE, }} \\
\quad 14,772.72)]\end{array}$ & $\begin{array}{l}\text { Attributable total operating costs, }(2000 \\
\text { USD), } \$ 46,133 \\
\text { (2019 USD, } \$ 69,083.62)\end{array}$ \\
\hline $\begin{array}{l}\text { Wilson } \\
(2014)^{44}\end{array}$ & $\begin{array}{l}\text { Age, sex, CVC type, total number of line-days, the number of times the CVC was } \\
\text { accessed by hospital personnel other than those on the pediatric hematology/ } \\
\text { oncology ward, number of blood cultures and the total number of excessive } \\
\text { blood cultures. }\end{array}$ & $\begin{array}{l}\text { Average, } \$ 107,007 \\
\text { (2019 USD, } \$ 122,824.60)\end{array}$ & $\begin{array}{l}\text { Average, } \$ 37,675 \\
\text { (2019 USD, } \$ 43,244.06)\end{array}$ & $\begin{array}{l}\text { Attributable (USD 2011), } \$ 69,332(95 \% \\
\quad \text { Cl, 35,144-103,521) } \\
{[2019 \text { USD, } \$ 79,580.54 \text { (SE, 20,021)] }}\end{array}$ \\
\hline
\end{tabular}

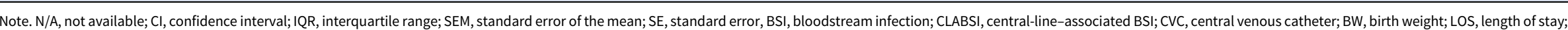
ICU, intensive care unit; NICU, neonatal ICU; PICU, pediatric ICU; BMTU, bone marrow transplantation unit. 
Table 4. Outcome Data of Included Studies With Regard to Mortality

\begin{tabular}{|c|c|c|c|c|}
\hline Author (Year) & Adjustment & Mortality, BSIs & Mortality, Non-BSIs & Attributable Mortality \\
\hline Duenas $(2011)^{30}$ & $\mathrm{~N} / \mathrm{A}$ & Crude, $25.0 \%$ & Crude, $13.6 \%$ & Crude extra, $11.4 \%$ \\
\hline Green $(2014)^{33}$ & N/A & $\begin{array}{l}\text { Crude, } 13 \text { of } 214 \\
(61 \text { per } 1,000)\end{array}$ & $\begin{array}{l}\text { Crude, } 157 \text { of } 333,391 \\
(0.5 \text { per } 1,000)\end{array}$ & N/A \\
\hline $\begin{array}{l}\text { Grisaru-Soen } \\
(2012)^{34}\end{array}$ & $\mathrm{~N} / \mathrm{A}$ & Crude, $6.9 \%$ & Crude, $3 \%$ & Crude attributable, $3.9 \%$ \\
\hline Gupta $(2011)^{35}$ & N/A & $\begin{array}{l}\text { HA-BSI crude fatality rate, } 55 \% \\
\text { CVC-BSIs crude fatality rate, } 55 \%\end{array}$ & $\begin{array}{l}\text { No HA-BSIs crude case-fatality } \\
\text { rate, } 30 \% \\
\text { No CVC-BSIs crude case-fatality } \\
\text { rate, } 41 \%\end{array}$ & $\begin{array}{l}\text { HA-BSI crude excess mortality, } 24 \% \\
\text { CVC-BSI crude excess mortality, } 14 \%\end{array}$ \\
\hline $\begin{array}{l}\text { Pessoa-Silva } \\
(2001)^{39}\end{array}$ & $\begin{array}{l}\text { Nasogastric/tracheal tube, lumbar/arterial puncture, } \\
\text { thoracic drains, blood and/or blood } \\
\text { components transfusion, umbilical catheter, } \\
\text { parenteral nutrition, duration of peripheral and central } \\
\text { venous catheters, and antibiotics }\end{array}$ & Crude, $32 \%$ & Crude, $8 \%$ & $\begin{array}{l}\text { Attributable mortality, } 24 \% \\
(95 \% \mathrm{Cl}, 9-39)\end{array}$ \\
\hline Rosenthal $(2014)^{41}$ & N/A & Pooled crude, $17.6 \%$ & Pooled crude, $6.2 \%$ & Pooled crude extra, $11.4 \%$ \\
\hline Rosenthal $(2012)^{40}$ & $\mathrm{~N} / \mathrm{A}$ & $\begin{array}{l}\text { Pooled crude, } 26.3 \% \\
(95 \% \mathrm{Cl}, 17.8-36.4)\end{array}$ & $\begin{array}{l}\text { Pooled crude, } 10 \% \\
(95 \% \mathrm{Cl}, 9-11.1)\end{array}$ & $\begin{array}{l}\text { Pooled crude excess, } 16.3 \% \\
(95 \% \mathrm{Cl}, 8.8-25.3)\end{array}$ \\
\hline Schwab $(2015)^{42}$ & $\begin{array}{l}\text { Case-control } \\
\text { BSI, adjusted by birth weight; } \\
\text { LC-BSI, no confounders/risk factors by the conditional } \\
\text { logistic regression }\end{array}$ & $\begin{array}{l}\text { Cohort (crude): } \\
\text { BSIs, 5.64\%; } \\
\text { LC-BSIs, } 10.47 \% \\
\text { Case-control study (crude): } \\
\text { BSIs, } 4.95 \% \text {; } \\
\text { LC-BSIs, } 9.53 \%\end{array}$ & $\begin{array}{l}\text { Cohort (crude): } \\
\text { No BSIs, } 6.81 \% ; \\
\text { No LC-BSIs, } 6.46 \% \\
\text { Case-control study (crude): } \\
\text { No BSIs, } 3.53 \% \text {; } \\
\text { No LC-BSIs, 3.04\% }\end{array}$ & $\begin{array}{l}\text { Cohort (crude), N/A } \\
\text { Case-control study (crude): } \\
\text { Attributable mortality of BSI, } 1.43 \% \\
\quad(95 \% \mathrm{Cl}, 0.69-2.17) \\
\text { Attributable mortality of LC-BSI, } 6.49 \% \\
\text { (95\% Cl, } 4.86-8.12)\end{array}$ \\
\hline Slonim $(2001)^{43}$ & $\mathrm{~N} / \mathrm{A}$ & Crude, $23.7 \%$ & Crude, $10.5 \%$ & Attributable crude, $13.1 \%$ \\
\hline
\end{tabular}

Note. N/A, not available; CI, confidence interval; BSI, bloodstream infection; HA-BSI, healthcare-acquired BSI; CVC-BSI, central venous catheter BSI; LC-BSI, laboratory confirmed BSI. 


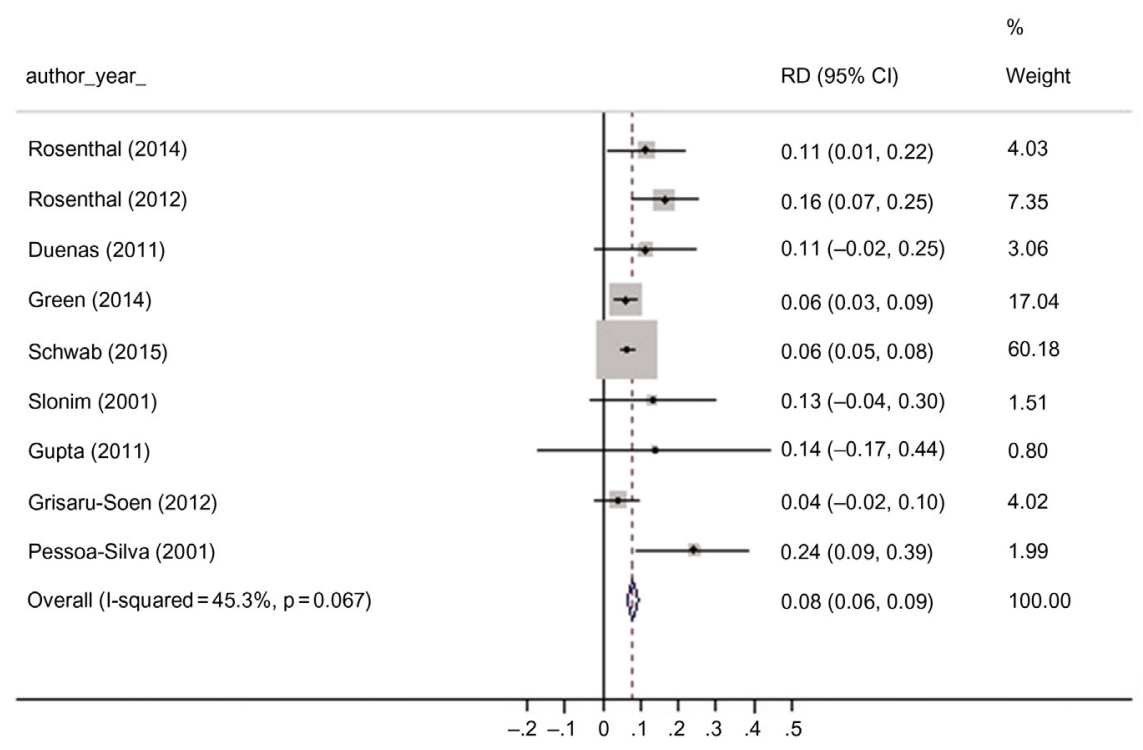

Fig. 4. Pooled overall attributable mortality rate, forest plot. Note. RD, risk difference.

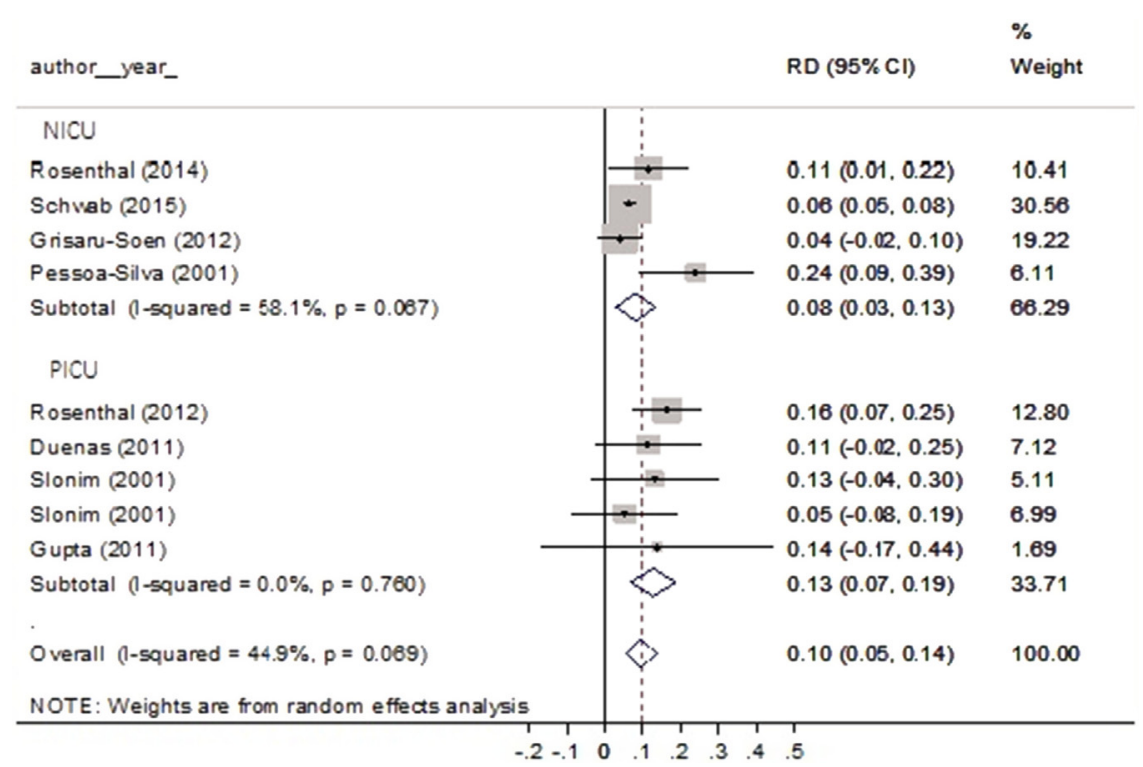

Fig. 5. Pooled attributable mortality rate by unit, forest plot. Note. RD, risk difference. although all of these BSIs were nosocomial, this could result in outcome differences. We tried to overcome this problem by conducting a subanalysis in studies assessing the attributable LOS and mortality of CLABSIs, and the outcomes were presented separately. The heterogeneity in the HAI definitions used by several authors in the literature is a major problem when trying to conduct a qualitative or quantitative synthesis of the available literature data on HAI outcomes.

In conclusion, HA-BSIs in children and neonates are associated with higher mortality, LOS, and healthcare costs than in children and neonates without HA-BSIs. This finding justifies and may enhance efforts to implement HA-BSI prevention strategies. Future research efforts could make better use of existing HAI definitions and evolving statistical methodologies, presenting more accurate, high-quality, and comparable outcome results globally.
Supplementary material. To view supplementary material for this article, please visit https://doi.org/10.1017/ice.2019.353

Financial support. This study was part of the doctoral thesis of the physician K.S., which was financed through a scholarship by the General Secretariat for Research and Technology (GSRT) and the Hellenic Foundation for Research and Innovation (HFRI).

Conflicts of interest. All authors report no conflicts of interest relevant to this article.

\section{References}

1. Zingg W, Holmes A, Dettenkofer M, et al. Hospital organization, management and structure for prevention of healthcare-associated infection: a systematic review and expert consensus. Lancet Infect Dis 2015;15:212-224. 
2. McClung L, Obasi C, Knobloch MJ, Safdar N. Healthcare worker perspectives of their motivation to reduce health care-associated infections $\mathrm{Am} \mathrm{J}$ Infect Control 2017;45:1064-1068.

3. Report on the burden of endemic healthcare-associated infection worldwide. World Health Organization website. https://apps.who.int/iris/bitstream/ handle/10665/80135/9789241501507_eng.pdf;jse, 2011. Accessed April 21, 2018.

4. Stone PW. Economic burden of healthcare-associated infections: an American perspective. Expert Rev Pharmacoecon Outcomes Res 2009;9: 417-422.

5. Annual epidemiological report on communicable diseases in Europe 2008: report on the state of communicable diseases in the EU and EEA/EFTA countries 2008. European Centre for Disease Prevention and Control website. https://www.ecdc.europa.eu/sites/portal/files/media/en/publications/ Publications/0812_SUR_Annual_Epidemiological_Report_2008.pdf, 2008. Accessed December 3, 2019.

6. Biwersi $\mathrm{C}$, Hepping $\mathrm{N}$, Bode $\mathrm{U}$, et al. Bloodstream infections in a German paediatric oncology unit: Prolongation of inpatient treatment and additional costs. Int J Hyg Environ Health 2009;212:541-546.

7. Zingg W, Hopkins S, Gayet-Ageron A, et al. Healthcare-associated infections in neonates, children, and adolescents: an analysis of paediatric data from the European Centre for Disease Prevention and Control pointprevalence survey. Lancet Infect Dis 2017;17:381-389.

8. Miliaraki M, Katzilakis N, Chranioti I, et al. Central-line-associated bloodstream infection in childhood malignancy: single-center experience. Pediatr Int 2017;59:769-775.

9. Gaur AH, Bundy DG, Werner EJ, et al. A prospective, holistic, multicenter approach to tracking and understanding bloodstream infections in pediatric hematology-oncology patients. Infect Control Hosp Epidemiol 2017;38: 690-696.

10. Umscheid CA, Mitchell MD, Doshi JA, Agarwal R, Williams K, Brennan PJ. Estimating the proportion of healthcare-associated infections that are reasonably preventable and the related mortality and costs. Infect Control Hosp Epidemiol 2011;32:101-114.

11. Mobley RE, Bizzarro MJ. Central-line-associated bloodstream infections in the NICU: Successes and controversies in the quest for zero. Semin Perinatol 2017;41:166-174.

12. The Matching Michigan Collaboration \& Writing Committee. 'Matching Michigan': a 2-year stepped interventional programme to minimize central venous catheter-blood stream infections in intensive care units in England. BMJ Qual Saf 2012;0:1-14.

13. Rallis D, Karagianni P, Papakotoula I, Nikolaidis N, Tsakalidis C. Significant reduction of central-line-associated bloodstream infection rates in a tertiary neonatal unit. Am J Infect Control 2016;44:485-487.

14. Johnson L, Grueber S, Schlotzhauer C, et al. A multifactorial action plan improves hand hygiene adherence and significantly reduces centralline-associated bloodstream infections. Am J Infect Control 2014;42: 1146-1151.

15. Bizzarro MJ, Sabo B, Noonan M, Bonfiglio M, Northrup V, Diefenbach K. A quality improvement initiative to reduce central-line-associated bloodstream infections in a neonatal intensive care unit. Infect Control Hosp Epidemiol 2010;31:241-248.

16. Sagana R, Hyzy RC. Achieving zero central-line-associated bloodstream infection rates in your intensive care unit. Crit Care Clin 2013;29:1-9.

17. Rosenthal VD, Al-Abdely HM, El-Kholi AA, et al. International Nosocomial Infection Control Consortium report, data summary of 50 countries for 2010-2015: device-associated module. Am J Infect Control 2016;44:1495-1504.

18. Leblebicioglu H, Erben N, Rosenthal VD, et al. International Nosocomial Infection Control Consortium (INICC) national report on device-associated infection rates in 19 cities of Turkey, data summary for 2003-2012. Ann Clin Microbiol Antimicrob 2014;13:51.

19. Venturini E, Montagnani C, Benni A, et al. Central-line-associated bloodstream infections in a tertiary-care children's university hospital: a prospective study. BMC Infect Dis. 2016;16:725.

20. De Angelis G, Murthy A, Beyersmann J, Harbarth S. Estimating the impact of healthcare-associated infections on length of stay and costs. Clin Microbiol Infect 2010;16:1729-1735.
21. Manoukian S, Stewart S, Dancer S, et al. Estimating excess length of stay due to healthcare-associated infections: a systematic review and meta-analysis of statistical methodology. J Hosp Infect 2018;100:222-235.

22. The PRISMA statement. PRISMA, transparent reporting of systematic reviews and meta-analyses. 2009. http://www.prisma-statement.org/. Accessed May 30, 2019.

23. Higgins JPT, Green S. General methods for Cochrane reviews. In: Higgins JPT, Thomas J, Chandler J, Cumpston M, Li T, Page MJ, Welch VA (editors). Cochrane Handbook for Systematic Reviews of Interventions. 2nd Edition. Chichester, UK: John Wiley \& Sons; 2019.

24. Aviles-Robles M, Ojha RP, Gonzalez M, et al. Bloodstream infections and inpatient length of stay among pediatric cancer patients with febrile neutropenia in Mexico City. Am J Infect Control 2014;42:1235-1237.

25. Allareddy V, Rampa S, Allareddy V. Hospital charges and length of stay associated with septicemia among children hospitalized for leukemia treatment in the United States. World J Pediatr 2012;8:222-228.

26. Dramowski A, Whitelaw A, Cotton MF. Burden, spectrum, and impact of healthcare-associated infection at a South African children's hospital. J Hosp Infect 2016;94:364-372.

27. Critical Appraisal Skills Programme Tool (CASP) for cohort and case-control studies. The Critical Appraisal Skills Programme website. https://caspuk.net/casp-tools-checklists/, 2018. Accessed December 3, 2019.

28. Aiken AM, Mturi N, Njuguna P, et al. Risk and causes of paediatric hospitalacquired bacteraemia in Kilifi District Hospital, Kenya: a prospective cohort study. Lancet 2011;378:2021-2027.

29. Atif ML, Sadaoui F, Bezzaoucha A, et al. Prolongation of hospital stay and additional costs due to nosocomial bloodstream infection in an Algerian neonatal care unit. Infect Control Hosp Epidemiol 2008;29:1066-1070.

30. Duenas L, Bran de Casares AC, Rosenthal VD, Machuca LJ. Device-associated infection rates in pediatric and neonatal intensive care units in El Salvador: Findings of the INICC. J Infect Dev Ctries 2011;5:445-451.

31. Elward AM, Hollenbeak CS, Warren DK, Fraser VJ. Attributable cost of nosocomial primary bloodstream infection in pediatric intensive care unit patients. Pediatrics 2005;115:868-872.

32. Goudie A, Dynan L, Brady PW, Rettiganti M. Attributable cost and length of stay for central-line-associated bloodstream infections. Pediatrics 2014;133: e1525-1532.

33. Green N, Johnson AP, Henderson KL, et al. Quantifying the burden of hospital-acquired bloodstream infection in children in England by estimating excess length of hospital stay and mortality using a multistate analysis of linked, routinely collected data. J Pediatr Infect Dis Soc 2015;4:305-312.

34. Grisaru-Soen G, Friedman T, Dollberg S, Mishali H, Carmeli Y. Late-onset bloodstream infections in preterm infants: a 2-year survey. Pediatr Int. 2012;54:748-753.

35. Gupta A, Kapil A, Lodha R, et al. Burden of healthcare-associated infections in a paediatric intensive care unit of a developing country: a single-centre experience using active surveillance. J Hosp Infect 2011;78:323-326.

36. Karagiannidou S, Zaoutis T, Maniadakis N, Papaevangelou V, Kourlaba G. Attributable length of stay and cost for pediatric and neonatal central lineassociated bloodstream infections in Greece. J Infect Public Health 2019;12: 372-379.

37. Navoa-Ng JA, Berba R, Galapia YA, et al. Device-associated infections rates in adult, pediatric, and neonatal intensive care units of hospitals in the Philippines: International Nosocomial Infection Control Consortium (INICC) findings. Am J Infect Control 2011;39:548-554.

38. Payne NR, Carpenter JH, Badger GJ, Horbar JD, Rogowski J. Marginal increase in cost and excess length of stay associated with nosocomial bloodstream infections in surviving very low birth weight infants. Pediatrics 2004;114:348-355.

39. Pessoa-Silva CL, Miyasaki CH, de Almeida MF, Kopelman BI, Raggio RL, Wey SB. Neonatal late-onset bloodstream infection: attributable mortality, excess of length of stay and risk factors. Eur J Epidemiol 2001;17:715-720.

40. Rosenthal VD, Jarvis WR, Jamulitrat S, et al. Socioeconomic impact on device-associated infections in pediatric intensive care units of $16 \mathrm{lim}$ ited-resource countries: International Nosocomial Infection Control Consortium findings. Pediatr Crit Care Med 2012;13:399-406.

41. Rosenthal VD, Maki DG, Mehta Y, et al. International Nosocomial Infection Control Consortiu (INICC) report, data summary of 43 countries for 20072012: device-associated module. Am J Infect Control 2014;42:942-956. 
42. Schwab F, Zibell R, Piening B, Geffers C, Gastmeier P. Mortality due to bloodstream infections and necrotizing enterocolitis in very low birth weight infants. Pediatr Infect Dis J 2015;34:235-240.

43. Slonim AD, Kurtines HC, Sprague BM, Singh N. The costs associated with nosocomial bloodstream infections in the pediatric intensive care unit. Pediatr Crit Care Med 2001;2:170-174.

44. Wilson MZ, Rafferty C, Deeter D, Comito MA, Hollenbeak CS. Attributable costs of central-line-associated bloodstream infections in a pediatric hematology/oncology population. Am J Infect Control 2014;42:1157-1160.

45. Garner JS, Jarvis WR, Emori TG, Horan TC, Hughes JM. CDC definitions for nosocomial infections, 1988. Am J Infect Control 1988;16: 128-140.

46. Horan TC, Andrus M, Dudeck MA. CDC/NHSN surveillance definition of health care-associated infection and criteria for specific types of infections in the acute care setting. Am J Infect Control 2008;36:309-332.

47. Central-line-associated bloodstream infection (CLABSI) event. Centers for Disease Control and Prevention website. www.cdc.gov/nhsn/pdfs/pscmanual/ 4psc_clabscurrent.pdf, 2014. Accessed December 3, 2019.
48. Vermont Oxford Network. Vermont Oxford Network Database Manual of Operations for Infants Born in 1998. Burlington, VT: Vermont Oxford Network; 1997.

49. Vermont Oxford Network. Vermont Oxford Network Database Manual of Operations for Infants Born in 1999. Burlington, VT: Vermont Oxford Network; 1998.

50. Nelson RE, Nelson SD, Khader K, et al. The magnitude of time-dependent bias in the estimation of excess length of stay attributable to healthcare-associated infections. Infect Control Hosp Epidemiol 2015;36:1089-1094.

51. Ziegler MJ, Pellegrini DC, Safdar N. Attributable mortality of central-lineassociated bloodstream infection: systematic review and meta-analysis. Infection 2015;43:29-36.

52. Siempos II, Kopterides P, Tsangaris I, Dimopoulou I, Armaganidis AE. Impact of catheter-related bloodstream infections on the mortality of critically ill patients: a meta-analysis. Crit Care Med 2009;37:2283-2289.

53. Zhang Y, Chen XL, Huang AW, et al. Mortality attributable to carbapenemresistant Pseudomonas aeruginosa bacteremia: a meta-analysis of cohort studies. Emerg Microbes Infect 2016;5:e27. doi: 10.1038/emi.2016.22. 